\title{
Robust Classification Method for Underwater Targets Using the Chaotic Features of the Flow Field
}

\author{
Xinghua Lin ${ }^{1}$, Jianguo $\mathrm{Wu}^{1, *}$ and Qing Qin ${ }^{2}$ \\ 1 School of Mechanical Engineering, Hebei University of Technology, Tianjin 300401, China; \\ sslxh2009@126.com \\ 2 China Automotive Technology and Research Center Co., Ltd, Tianjin 300300, China; sxyqqq@sina.com \\ * Correspondence: wujianguo@hebut.edu.cn
}

Received: 17 January 2020; Accepted: 10 February 2020; Published: 12 February 2020

check for updates

\begin{abstract}
Fish can sense their surrounding environment by their lateral line system (LLS). In order to understand the extent to which information can be derived via LLS and to improve the adaptive ability of autonomous underwater vehicles (AUVs), a novel strategy is presented, which directly uses the information of the flow field to distinguish the object obstacle. The flow fields around different targets are obtained by the numerical method, and the pressure signal on the virtual lateral line is studied based on the chaos theory and fast Fourier transform (FFT). The compounded parametric features, including the chaotic features (CF) and the power spectrum density (PSD), which is named CF-PSD, are used to recognize the kinds of obstacles. During the research of CF, the largest Lyapunov exponent (LLE), saturated correlation dimension (SCD), and Kolmogorov entropy (KE) are taken into account, and PSD features include the number, amplitude, and position of wave crests. A two-step support vector machine (SVM) is built and used to classify the shapes and incidence angles based on the CF-PSD. It is demonstrated that the flow fields around triangular and square targets are chaotic systems, and the new findings indicate that the object obstacle can be recognized directly based on the information of the flow field, and the consideration of a parametric feature extraction method (CF-PSD) results in considerably higher classification success.
\end{abstract}

Keywords: object obstacle recognition; chaotic theory; flow sensing; underwater robot; SVM

\section{Introduction}

Vision and sonar are the primary sensory systems available to autonomous underwater vehicles (AUVs) for navigation [1-4]. However, because there are many obstacles near shore and the turbid environment is problematic, vision and sonar systems have many limitations for AUV operation. For example, sonar frequently produces false positives due to the multiple targets and relatively shallow water depth. Similarly, the function of the vision system is directly limited by the reduction of the visibility range due to the turbidity of water $[5,6]$. In addition to the difficulty in functionality, both vision and sonar systems require the acoustic or light energy to be sent into the fluid incessantly; this is named the active sensing system. This leads to substantial power consumption on platforms. Due to these limitations, AUVs are rarely used in the cluttered environment. In order to overcome these limitations, the question of improving the sensing method and adaptive ability is of particular interest to AUVs.

According to the research of biologists, many behaviors of fish are associated with their lateral line system (LLS), which is a versatile sensor organ [7]. Although it is unknown what specific information about stimuli is obtained by fish, the information can help fish to identify objects, track prey, and conserve energy while swimming in wakes [8-10]. Since LLS does not use light, the turbid environment has no affection on its function. LLS only focuses on the objects necessary for immediate navigation, and 
complicated structures not in the vicinity are disregarded. In other words, LLS naturally emphasizes the closest stimuli, providing both advantages and limitations. Besides, LLS is a passive system, resulting in its power consumption being more than an order of magnitude smaller than that of traditional systems [11]. This can provide detailed aid in navigation with little additional cost. Based on the bionic principle, if this sensory system can be translated to AUVs, it would provide substantial benefits in maneuvering and object detection in difficult environments. In recent years, there has been increasing interest in flow sensing. The research can be divided into two parts. One is the engineering design of sensors based on setting nature as a model, which is called biomimetics [12-14], and the other one is the hydrodynamic mechanism of LLS [15-18].

Biomimetics focuses on natural sensors, which have evolved over billions of years, developing extreme efficiency, compactness, high responsivity, and throughput. Engineers employ a variety of sensing principles to imitate superficial neuromasts and canal neuromasts, such as the piezoresistive [19], capacitive [20], thermal [21], magnetic [22], piezoelectric [23], and optical [24] techniques. However, there is currently no analog to the sensor for AUV, because the type of processing and information extracted by the fish has yet to be discovered. Hydrodynamics mainly focuses on the detection and localization of the dipole source [25-27]. One main way is the detection of Karman vortex streets (KVS), such as the exact location, shedding frequency, and fluctuation range of a vortex [28,29]. Another technique is the study of detection algorithms and nonlinear dynamic models, which are based on the neural network scheme and potential flow theory [30-34]. However, the detection of KVS only focuses on the local information of the flow field, and the environment is different to the natural underwater world. The role of flow measurements in fish responses is also unclear. Without understanding the global information that can be derived via the lateral line organ, it is impossible to transfer LLS to man-made vehicles.

The global information measurements in real systems are subject to uncertainties, which can complicate the obstacle recognition problem. To overcome this problem, obstacle recognition can be done based on nonlinear dynamics. Many existing features use the nonlinear characteristics of the flow field to recognize the obstacles. In this situation, the flow sensing problem becomes a problem of nonlinear detection. As is known to all, due to the turbulent wakes, downstream bluff-bodies can be very complex, and strongly intermittent behaviors are produced by large scale and small-scale coherent structures. In the study of automotive aerodynamics, chaotic theory has been used to study the dynamics of a 3D bimodal turbulent wake downstream of a square-back Ahmed body, and the low frequency dynamics of a turbulent 3D wake demonstrated that this is not a purely stochastic process but, rather, a weakly chaotic process exhibiting strange attractors [35]. A synchronization algorithm which uses a hydrodynamic analogy based on the transport of the properties of a particle convected in a fluid flow field was provided by Yechiel Crispin, and the synchronization algorithm was applied to two chaotic systems with variable parameters [36]. Inspired by this, the nonlinear characteristics of flow field can be obtained in the framework of chaotic systems theory.

Regarding the flow sensing of object obstacles, two aspects are the most interesting: what the object obstacle is and where it is. In our previous works, the location method was introduced [37]. In the following text, a new flow sensing method for target recognition is presented. We first show how the flow field around different obstacles can be characterized by the chaotic features (CF). In particular, $\mathrm{CF}$ includes the largest Lyapunov exponent, the correlation dimension, and the Kolmogorov entropy of the pressure signal time series. The relationship between CF and object shapes is considered primarily, and the family of the flow field to which it belongs is evaluated. After recovering the chaotic pattern, the inner characteristics of the pressure signal are analyzed more thoroughly based on the fast Fourier transform (FFT). The power spectrum density (PSD) is used to distinguish the incidence angle of an obstacle with the same shape. A complete recognizing technique that only uses flow pressure data as the input is provided. In addition, the relationship between the compounded parametric features CF-PSD and the estimation accuracy is examined by the support vector machine (SVM). The results illustrate that the proposed method achieves better detection performance. 
The paper is organized as follows. In Section 2, the background of the physical model and numerical method is described, and then the validation of the numerical results is strengthened by previous results. The signal obtaining method and the pressure signal around different targets are shown in Section 2. In Section 3, the novel target recognition strategy is described, including the recognition of shapes and incidence angles, which depend on CF and PSD, respectively. The support vector machine (SVM) is selected as the classifier in Section 4, and numerical experiments are taken for the evaluation of the proposed strategy. In the experiments, three types of underwater target are analyzed and classified under different incidence angles. Finally, concluding remarks are provided in Section 5.

\section{Signal Acquisition and Processing Methods}

\subsection{Physical Model}

The flow fields around targets with different shapes and incidence angles are computed based on computational fluid dynamics (CFD). Three types of underwater targets are analyzed, including circular, triangular, and square. We illustrate an example of a square target, as seen in Figure 1; its side length is $H$, and the incidence angle is $\theta$. The computational domain $\Omega$ is located in a Cartesian coordinate with a range of $30 \mathrm{H} \times 10 \mathrm{H}$. The virtual lateral line is under the center of the targets with a distance of $d$. The sensors are located at $\left(x_{i}, y_{i}\right)$, and their range is shown in Equation (1). The disturbance of sensors to the flow field is negligible.

$$
\left\{\begin{array}{l}
-10 H \leq x_{i} \leq 10 H \\
y_{i}=-d
\end{array}\right.
$$

where $H$ is the characteristic length of the target, and $d$ is the detective distance.

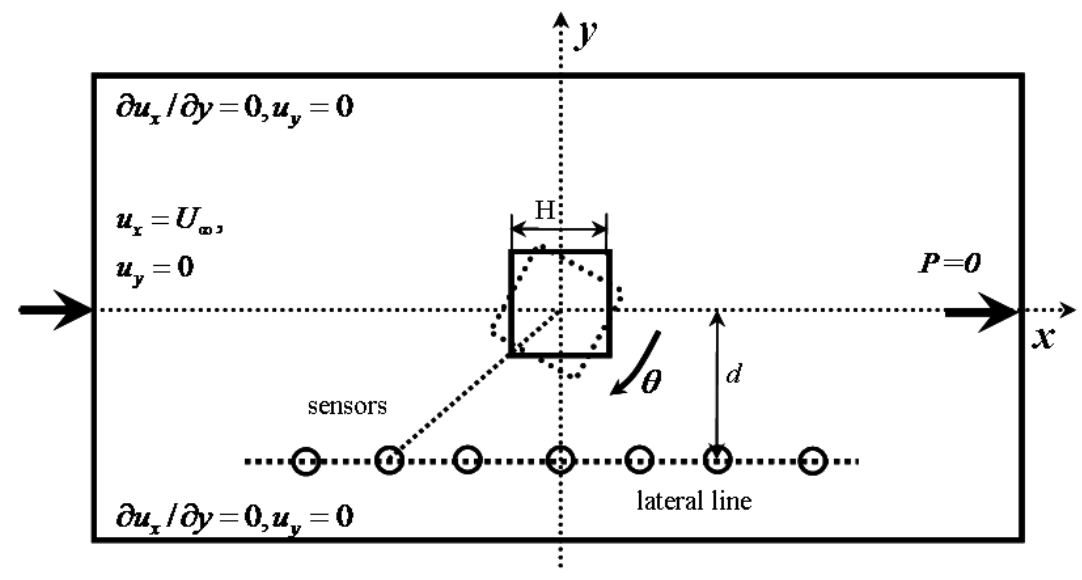

Figure 1. Schematic diagram of the computational domain for a square target.

As with our previous works [37], the velocity-inlet and pressure-outlet boundaries are used to create a uniform flow environment. The lateral boundaries are described by the symmetry condition, and the surface of the targets is seen as a no-slip boundary. A grid increasing function is built to mesh the computational domain $\Omega$, which is shown in Equation (2). The surface of the target is meshed with $\chi_{\min }$, and the further domain is meshed with $\chi(i)$. The ratio of the grid size between two adjacent layers is $\delta$, and the outside surface of the computational domain $\Omega$ is meshed $\chi_{\max }$. The square target is used to study the grid independence, and three cases with different minimum sizes $\chi_{\min }$ are taken into account. When $\delta$ is 1.2, the Strouhal number $S_{t}$ and its percentage changes are shown in Table 1. It is observed that there is no significant change between Grid 1 and Grid 2. However, the required computer resource of Grid 1 is larger than that of Grid 2. Therefore, Grid 2 is used in the investigations presented.

$$
\left\{\begin{array}{l}
\chi(i)=\delta \cdot \chi_{\min } \\
\chi_{\min } \leq \chi(i) \leq \chi_{\max }
\end{array} .\right.
$$


Table 1. Analysis of grid independence.

\begin{tabular}{ccccc}
\hline Case & $\chi_{\min }$ & Number of Elements & $S_{t}$ & Percentage Changes/\% \\
\hline Grid 1 & $0.0001 H$ & $8.27 \times 10^{6}$ & 0.139 & $\backslash$ \\
Grid 2 & $0.001 H$ & $6.26 \times 10^{5}$ & 0.142 & 2.1 \\
Grid 3 & $0.01 H$ & $4.82 \times 10^{4}$ & 0.153 & 7.2 \\
\hline
\end{tabular}

\subsection{Numerical Model and Validation}

The flow field around different targets is solved based on the two-step Taylor-characteristic-based Galerkin method (TCBG), which was introduced by Bao et al. [38]. The solution method is shown in Equation (3) to Equation (5). There are three steps in the discretizing process: the half-time velocity $u_{i}^{n+1 / 2}$ can be obtained from Equation (3) firstly, and then from Equation (5), the relationship of $p^{n+1}$ and $u_{i}^{n+1}$ can be obtained. Finally, Equation (4) can be solved to get the full-time velocity $u_{i}^{n+1}$ and pressure $p^{n+1}$ based on the above relationship.

$$
\begin{gathered}
u_{i}^{n+1 / 2}=u_{i}^{n}-\frac{\Delta t}{2}\left(u_{j}^{n} \frac{\partial u_{i}^{n}}{\partial x_{j}}+\frac{\partial p^{n}}{\partial x_{i}}-\frac{1}{\operatorname{Re}} \frac{\partial \tau_{i j}^{n}}{\partial x_{j}}\right)+\frac{\Delta t^{2}}{8} u_{k}^{n} \frac{\partial}{\partial x_{k}}\left(u_{j}^{n} \frac{\partial u_{i}^{n}}{\partial x_{i}}+2\left(\frac{\partial p^{n}}{\partial x_{j}}-\frac{1}{\operatorname{Re}} \frac{\partial \tau_{i j}^{n}}{\partial x_{j}}\right)\right) \\
\left.u_{i}^{n+1}=u_{i}^{n}-\Delta t\left(u_{j}^{n+1 / 2} \frac{\partial u_{i}^{n}}{\partial x_{j}}+\frac{\partial p^{n+1}}{\partial x_{i}}-\frac{1}{\operatorname{Re}} \frac{\partial \tau_{i j}^{n+1 / 2}}{\partial x_{j}}\right)+\frac{\Delta t^{2}}{2} u_{k}^{n+1 / 2} \frac{\partial}{\partial x_{k}}\left(u_{j}^{n+1 / 2} \frac{\partial u_{i}^{n}}{\partial x_{i}}+\frac{\partial p^{n+1}}{\partial x_{i}}-\frac{1}{\operatorname{Re}} \frac{\partial \tau_{i j}^{n+1 / 2}}{\partial x_{j}}\right)\right) \\
\frac{\partial^{2} p^{n+1}}{\partial x_{i} \partial x_{i}}=\frac{1}{\Delta t} \frac{\partial}{\partial x_{i}}\left(u_{i}^{n}-u_{i}^{n+1}\right)-\frac{\partial}{\partial x_{i}}\left(u_{j}^{n+1 / 2} \frac{\partial u_{i}^{n+1 / 2}}{\partial x_{j}}-\frac{1}{\operatorname{Re}} \frac{\partial \tau_{i j}^{n+1 / 2}}{\partial x_{j}}\right)
\end{gathered}
$$

where $u_{i}$ is the $i$-component velocity, $\rho$ is the water density, $p$ is the pressure, $\tau_{i j}$ is the deviatoric stresses, Re is the Reynolds number, and $n, n+1 / 2$, and $n+1$ denote the time points of $t_{n}, t_{n+1 / 2}$, and $t_{n+1}$, respectively.

The accuracy of the TCBG method was validated by Bao et al. [38]. In this section, the numerical algorithm is further demonstrated by previous results $[37,39,40]$ when it is used to simulate the flow past a square target with different incidence angles. As seen in Figure 2, the Strouhal numbers $S_{t}$ are in accordance with previous reports, which implies that there is reasonable agreement between our numerical results and previous data.

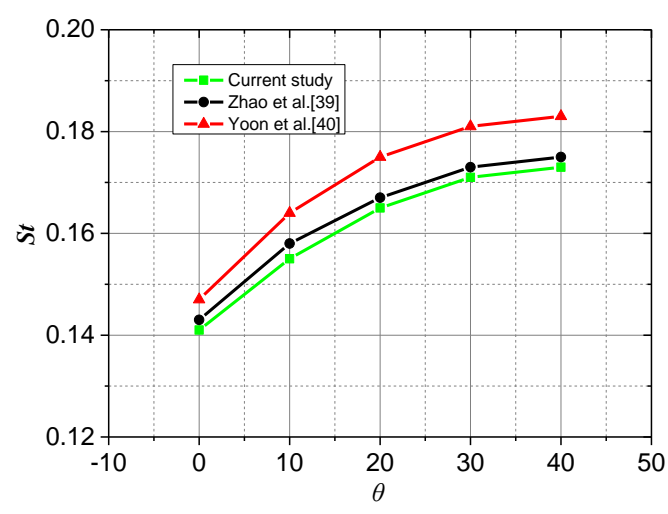

Figure 2. Simulated results compared with previous results.

\subsection{Signal Fusion Method}

A single sensor cannot respond to the flow structure accurately. Thus, multi-sensors are set at the lateral line, and the signal of each sensor is merged with a weighting fusion algorithm to get the final signal time series of the flow structure. The position of the sensors and the signal amplitude are 
considered in the algorithm. Thus, the position coefficient $S_{i}$ and the amplitude coefficient $A_{i}$ form the instantaneous weight coefficient $K_{i}$, which is expressed by Equation (6).

$$
\left\{\begin{array}{l}
K_{i}=S_{i}+A_{i} \\
S_{i}=\frac{\varepsilon \sin \alpha_{i}}{\sum_{i=0}^{N} \sin \alpha_{i}} \\
A_{i}=\frac{(1-\varepsilon) A_{m}\left(s_{i}\right)}{\sum_{i=0}^{N} A_{m}\left(s_{i}\right)} \\
c_{p}(t)=\sum_{i=0}^{N} K_{i} S_{i}(t)
\end{array}\right.
$$

where $\varepsilon$ is the weight of position coefficient $S_{i}$. If $\varepsilon$ becomes larger, this implies that the influence of position is more obvious. $A_{m}\left(s_{i}\right)$ is the amplitude of a signal collected by sensor $i$.

The flow fields around circular, triangular, and square targets are studied, respectively, based on CFD. The lateral line is set at the position of $d=2 H$, and the pressure signal $C_{p}$ is collected and shown in Equation (7).

$$
C_{p}=\frac{p_{i}-p_{\infty}}{\frac{1}{2} \rho V^{2}}
$$

where $p_{i}$ is the pressure at the measuring point, $p_{\infty}$ is the pressure at far field, which is 101,325 Pa in this paper, $\rho$ is the density of water, and $V$ is the inlet velocity.

There are 21 measuring points on the lateral line, and their signals are weighted and fused to obtain the time series, which is shown in the Figure 3. As seen in Figure 3, the pressure signal for the circular target is periodic after five seconds, and the signals for the triangular target and square target are aperiodic. The inner characteristics of the flow field are analyzed by chaotic theory and fast Fourier transform in the following section.
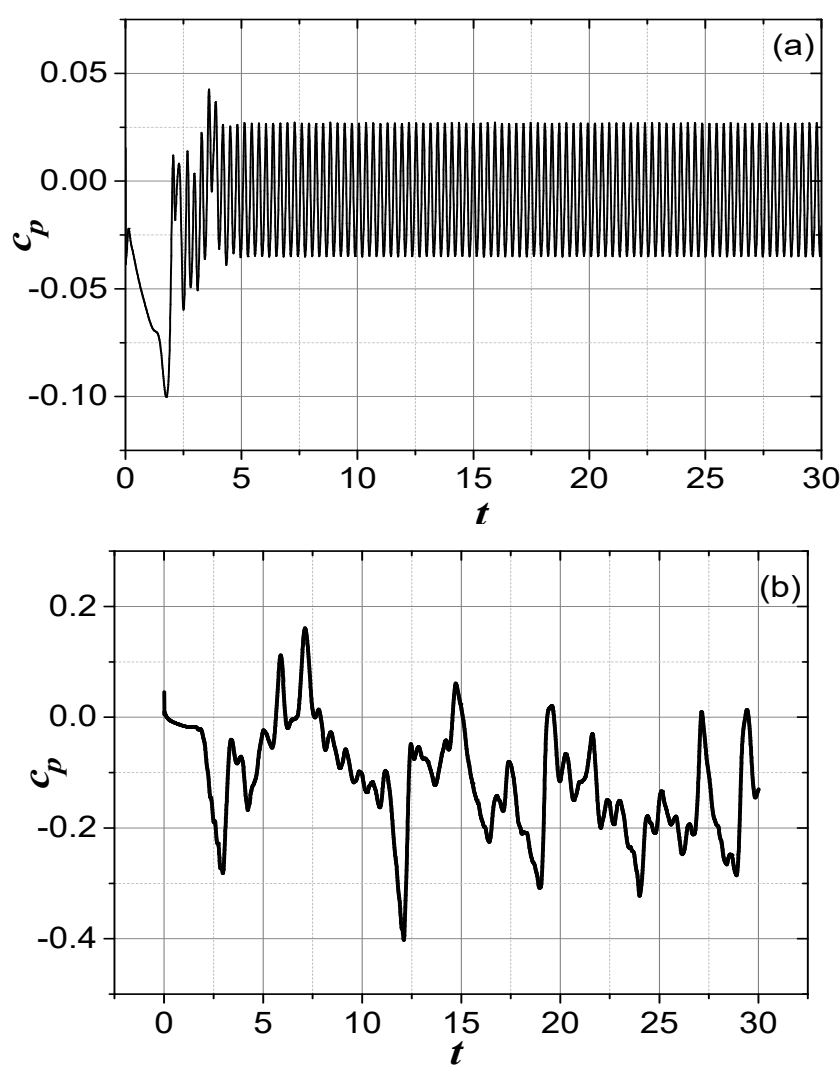

Figure 3. Cont. 


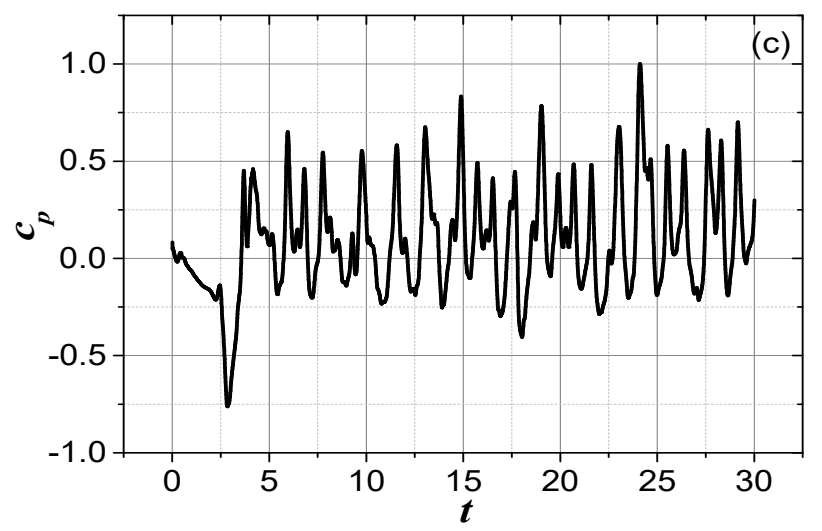

Figure 3. Waveforms of the three types of signals ((a) circular target; (b) triangular target; (c) square target).

\section{Target Recognition Strategy}

\subsection{Feature Selection for Shape Classification}

\subsubsection{Phase Space Reconstruction}

The dynamic characteristics of a times series can be revealed by the chaos attractor, and we can use it to estimate whether the system is chaotic or stochastic. Therefore, attractor reconstruction is a key step in chaotic time series analyses. In order to reveal its chaos attractor, a high-dimensional space is built to express the time series of the pressure signal, a method which was proposed by Takens [41]. For a given time series $\left\{c_{p}(t)\right\}$, the phase space can be expressed as Equation (8) with an optimal delay time $\tau$ and an optimal embedding dimension $m$.

$$
C_{p}\left(t_{i}\right)=\left[c_{p}\left(t_{i}\right), c_{p}\left(t_{i}+\tau\right), c_{p}\left(t_{i}+2 \tau\right), \ldots, c_{p}\left(t_{i}+(m-1) \tau\right], i=1,2, \ldots, N-(m-1) \tau,\right.
$$

where $\tau$ is the delay time, $m$ is the embedding dimension, and $N$ is the length of the phase space.

The C-C method is used to select the optimal delay time $\tau$ and the optimal embedding dimension $m$, a method which was proposed by Kim H.S. et al. [42]. The method has a strong ability to resist noise with little calculation cost, and the nonlinear characteristics of the time series can be kept. If the parameters meet the requirements of Equation (9), the correlation of pressure vibration intensity series can be reflected by $\bar{S}(t)$ and $\Delta \bar{S}(t)$, which is shown in Equation (10). The solving progress is as follows:

1. The optimal delay time $\tau$ will correspond to the first local minimum value times of $\Delta \bar{S}(t)$;

2. The embedding dimension $m$ can be obtained from the delay time window $\tau_{m}$, where $\tau_{m}=$ $(m-1) \cdot \tau$, and $\tau_{m}$ correspond to the minimum value of the quantity $S_{c o r}(t)$.

$$
\begin{gathered}
\left\{\begin{array}{l}
2 \leq m \leq 5 ; \\
\frac{1}{2} \sigma \leq r \leq 2 \sigma ; \\
N \geq 500
\end{array}\right. \\
\left\{\begin{array}{l}
\bar{S}(t)=\frac{1}{16} \sum_{m=2}^{5} \sum_{j=1}^{4} S\left(m, r_{j}, t\right) ; \\
\Delta \bar{S}(t)=\frac{1}{4} \sum_{m=2}^{5} \Delta S(m, t) ; \\
S_{c o r}(t)=\Delta \bar{S}(t)+|\Delta \bar{S}(t)| ; \\
S\left(m, r_{j}, t\right)=\frac{1}{t} \sum_{S=1}^{t}\left[C_{s}\left(m, r_{j}, t\right)-C_{s}^{m}\left(1, r_{j}, t\right)\right] ; \\
\Delta S(m, t)=\max \left\{S\left(m, r_{j}, t\right)\right\}-\min \left\{S\left(m, r_{j}, t\right)\right\}
\end{array}\right.
\end{gathered}
$$

where $r$ is the radius of time series, and $\sigma$ is the time series standard deviation. 


\subsubsection{The Largest Lyapunov Exponent (LLE)}

The Lyapunov exponent $\lambda$ is an important parameter that is used to characterize chaotic time series, where the exponential rates of divergence or convergence of nearby trajectories in phase space can be reflected by $\lambda$. If LLE is positive, this implies that the phase plane trajectory is chaotic $[43,44]$. The Wolf algorithm is used to calculate the LLE [45], which has been proven to be more robust than the least-squares method [46]. In the reconstructed phase space, two adjacent points $C_{p, n 1}$ and $C_{p, n 1}+\delta C_{p}$ are selected to calculate the distance $\delta C_{p}$ between those two points. Given a residual parameter $\zeta$, if the distance is outside of $\zeta$ after one time iteration, new neighbor points will be selected repeatedly until the fiducial trajectory has traversed the entire data series. The Lyapunov exponent can be obtained from Equation (11), and LLE is the largest one $\lambda$.

$$
\left\{\begin{array}{l}
\delta C_{p}(n)=\frac{d f^{n}\left(C_{p, n 1}\right)}{d C_{p}} \cdot \delta C_{p, n 1}=e^{\lambda \cdot n} \cdot \delta C_{p, n 1} \\
\lambda=\lim _{n \rightarrow \infty} \frac{1}{n} \sum_{i=0}^{n-1} \ln \left|\frac{d f\left(C_{p}\right)}{d C_{p}}\right|_{C_{p}=C_{p}(i)}
\end{array} .\right.
$$

The LLEs of the pressure signal time series around the circular target, triangular target and square target are calculated using the above method. As seen in Figure 4, linear regression is conducted to get $\lambda$ in the range of time steps $i$ from 15 to 25, and the results are shown in Figure 5. From Figure 5, the LLE of the flow field around the circular target is negative, which implies that the time series is regular. However, the results of LLE around the triangular target and square target are positive, which implies their time series are chaotic sequences.

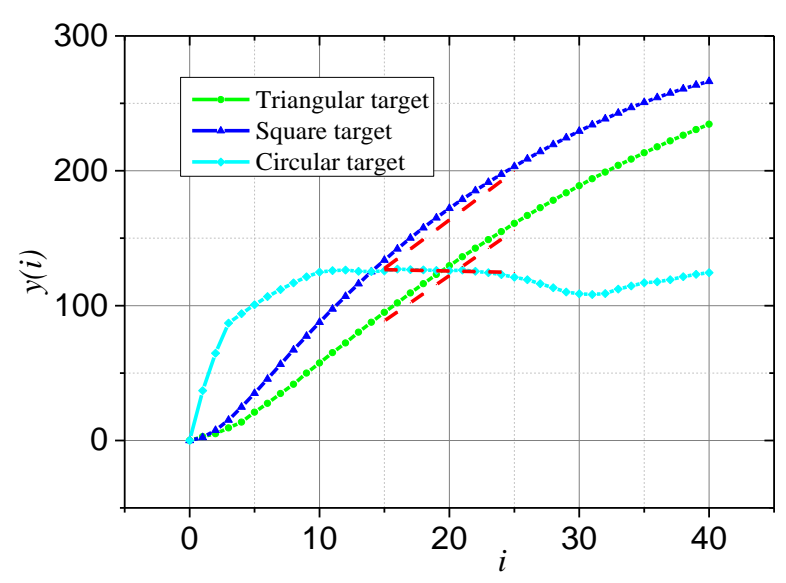

Figure 4. Relation curve between $y(i)$ and $i$ for different targets.

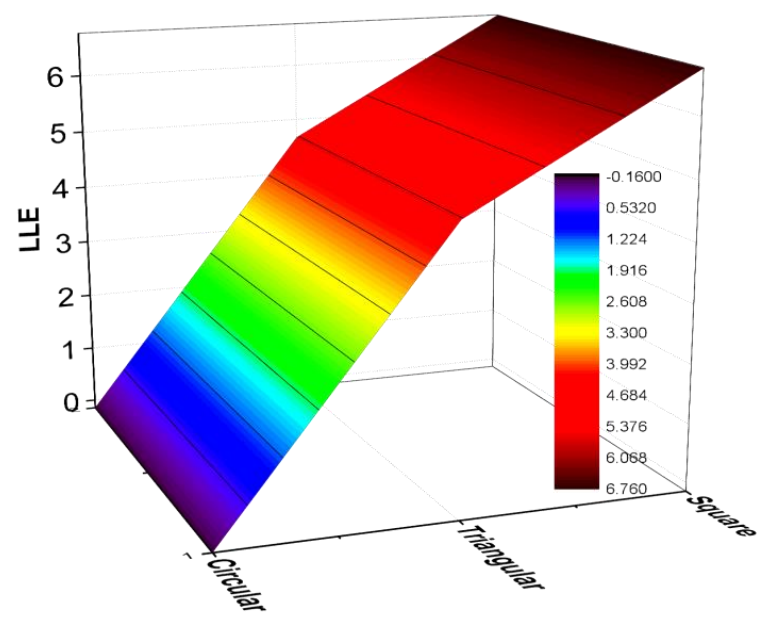

Figure 5. Largest Lyapunov exponent (LLE) of pressure signal time series in different flow fields. 
Another interesting result is that the LLEs of different targets have no overlap. There are obvious characteristic features. This indicates that the shape of underwater targets can be identified by LLE.

\subsubsection{The Saturated Correlation Dimension (SCD)}

The fractal dimension of a reconstruction attractor in phase space can be reflected by the SCD, which is calculated by the Grassberger-Procaccia (G-P) algorithm in this paper [47,48]. According to the algorithm, SCD can be derived from the correlation integral function, which is defined as Equation (12) in the reconstructed phase space.

$$
\left\{\begin{array}{l}
C(\tau)=\frac{2}{M(M-1)} \sum_{i=1}^{M} \sum_{j=i+1}^{M} \Theta\left(\tau-d_{i j}\right), r>0 \\
d_{i j}=\left|C_{p}\left(t_{i}\right)-C_{p,}\left(t_{j}\right)\right| \\
M=N-(m-1) \tau \\
\Theta(x)=\left\{\begin{array}{l}
0, x \leq 0 \\
1, x>0
\end{array}\right.
\end{array},\right.
$$

where $C(\tau)$ is the correlation integral function, and $\Theta(x)$ is the Heaviside function.

The SCD can be established by the asymptotic curve of $\ln C(\tau) \sim \ln (\tau)$. There is a linear relationship between them, which is shown in Equation (13). For the pressure signal of the circular target, the correlation integral function in logarithmic scale is shown in Figure 6. As seen in Figure 6, the power law approximation is $\tau^{1.0}$, which means that SCD is 1.0. The embedding dimension $m$ can be obtained by SCD based on the Equation (14), and the embedding dimension is 3 .

$$
\begin{gathered}
S C D=\lim _{r \rightarrow 0 N \rightarrow \infty} \frac{\ln (C(\tau))}{\ln (\tau)}, \\
m \geq 2 S C D+1
\end{gathered}
$$

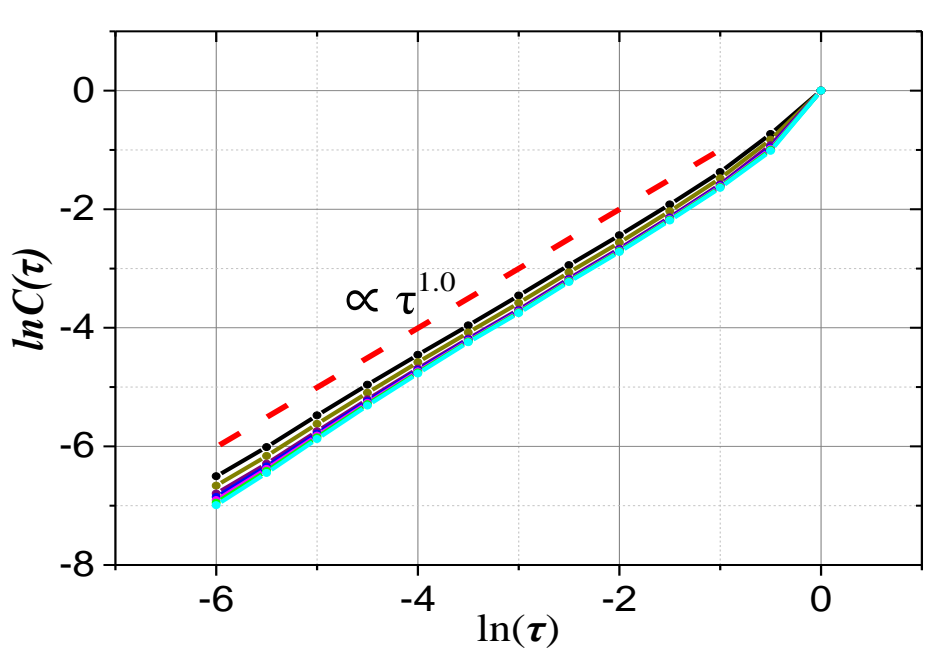

Figure 6. Relation curve between $\ln C(\tau)$ and $\ln (\tau)$ for the circular target.

Using the same method, the SCD values of triangular and square targets can be obtained. The SCD increases with an increase in the embedding dimension $m$, which is shown in Figures 7 and 8 . In Figure 7 , the curve region reaches a saturated state gradually as the embedding dimension increases, and the convergence value SCD is 4.715 , which means that the embedding dimension is 11 . For the pressure signal of the square target, the correlation dimension is 4.826 and the embedding dimension is 11 . This means that the pressure intensity time series can be completely revealed in 11-dimensional space. 


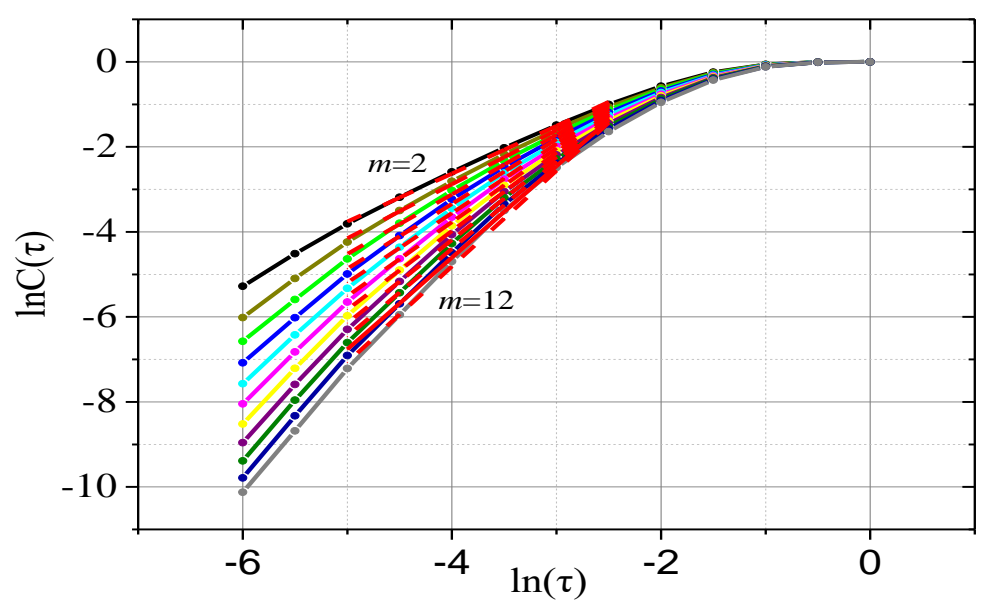

Figure 7. Relation curve between $\ln C(\tau)$ and $\ln (\tau)$ for the triangular target.

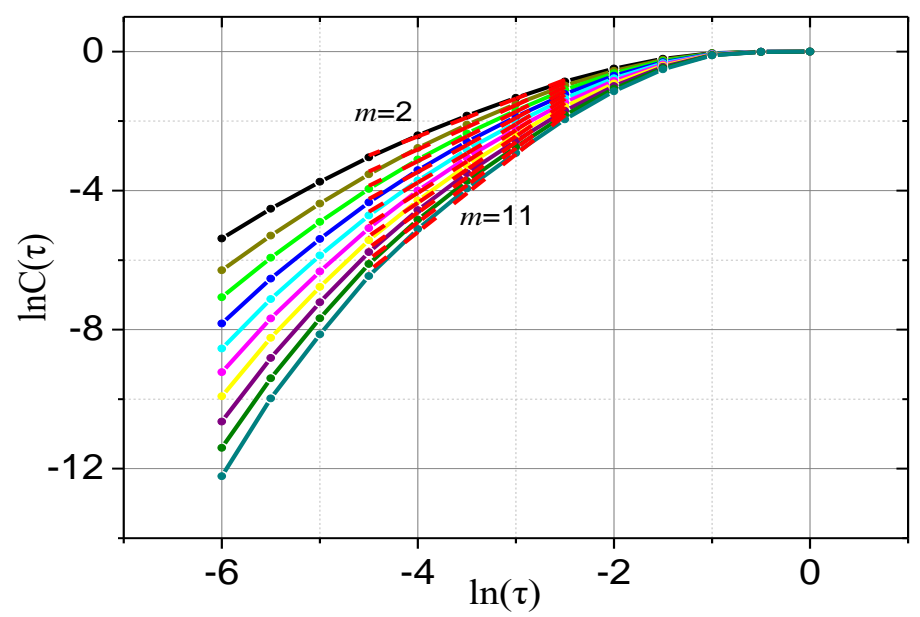

Figure 8. Relation curve between $\ln C(\tau)$ and $\ln (\tau)$ for the square target.

The most interesting result is that the SCD values of different targets also have no overlap, which is shown in Figure 9. This result implies that the SCD can be used to distinguish the shapes of targets.

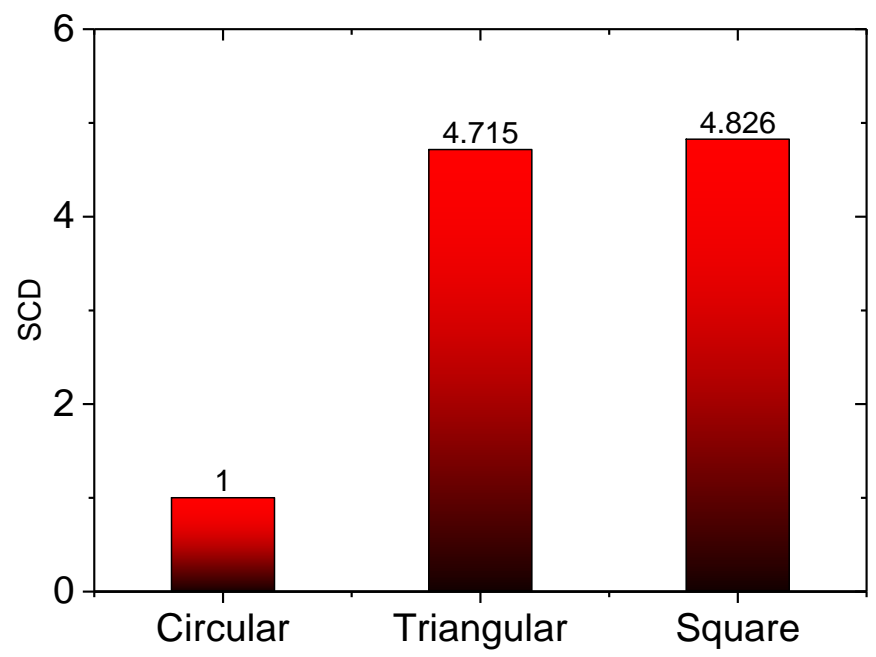

Figure 9. Saturated Correlation Dimension (SCD) of pressure signal time series in different flow fields. 


\subsubsection{The Kolmogorov Entropy (KE)}

The Kolmogorov entropy is an important parameter in a chaotic system. It can describe the rate of information loss and is often used to quantify the unpredictability of a chaotic system. In order to measure the chaotic systems, if the embedding dimension increases from $m$ to $m+d$, the KE can be obtained based on the G-P algorithm, as shown in Equation (15).

$$
K=\lim _{r \rightarrow 0 m \rightarrow \infty} \lim _{m \rightarrow} \frac{1}{d \tau} \ln \frac{C_{m}(r)}{C_{m+d}(r)} .
$$

Based on the above analysis, the KE of a fully predictable system is zero; for a chaotic system, KE is a positive and finite number, and KE has no limitation for a stochastic system. As seen in Figure 6, the embedding dimension has no influence on its SCD in the flow field around the circular target, which implies that $K_{\text {cir }}=0$. As seen in Figure 10, for the triangular target and square target, KE will decrease sharply with an increase in the embedding dimension $m$ at a small embedding dimension. When the embedding dimension is high enough, KE tends to be a common value. For the triangular target, the common value is $K_{t r i}=4.431$ and for the square target, $K_{s q u}=4.648$. This indicates that another chaotic feature, $\mathrm{KE}$, can be used to distinguish the shapes of targets.

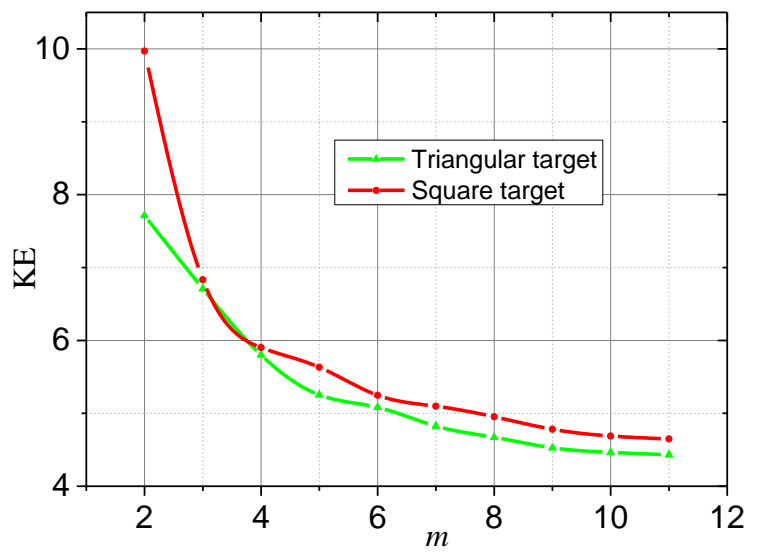

Figure 10. Relation curve between the Kolmogorov entropy (KE) and embedding dimension for the triangular and square targets.

\subsection{Feature Selection for Incidence Angle Classification}

\subsubsection{Analysis of the Chaotic Features}

The square target is chosen for analysis with different incidence angles $(\theta \in[10,40])$. Their chaotic features are analyzed based on the above method. The LLE of the square target with different incidence angles is shown in Figure 11. From Figure 11, we can see that at small incidence angles, the LLE will decrease gradually with an increase in the incidence angle. However, LLE increases sharply when the incidence angle is $40^{\circ}$. The result is not wanted, because it implies that the LLE has no monotonicity with the change in the incidence angle, and we cannot use LLE to distinguish the status.

The same analysis is employed to discuss the SCD and the KE. The results are shown in Figures 12 and 13. Disappointingly, they have overlap when the incidence angles are different, which implies that they are also not monotonic. These results prove that the CF cannot be used as features to recognize the incidence angles of one target. We should find new features to complete the goal. 


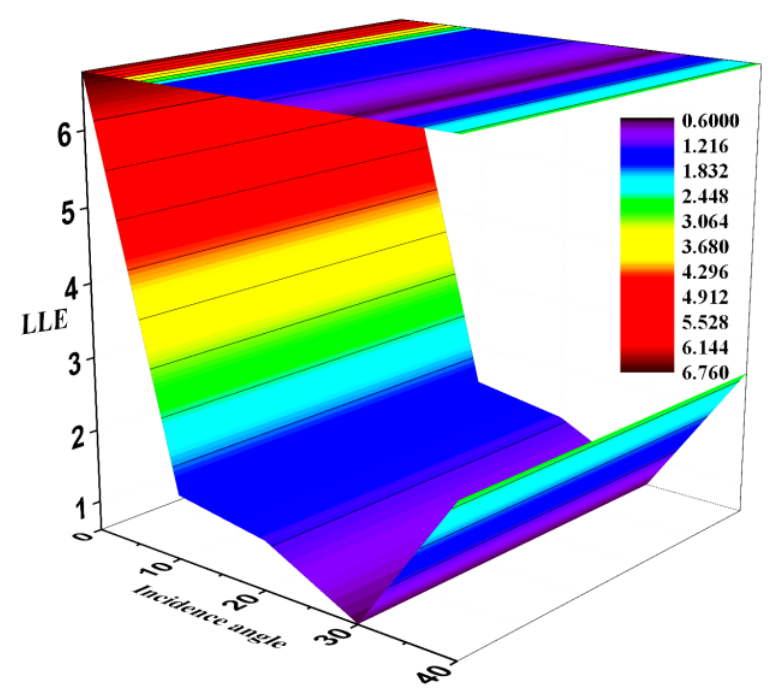

Figure 11. LLE of the pressure signal time series at different incidence angles.

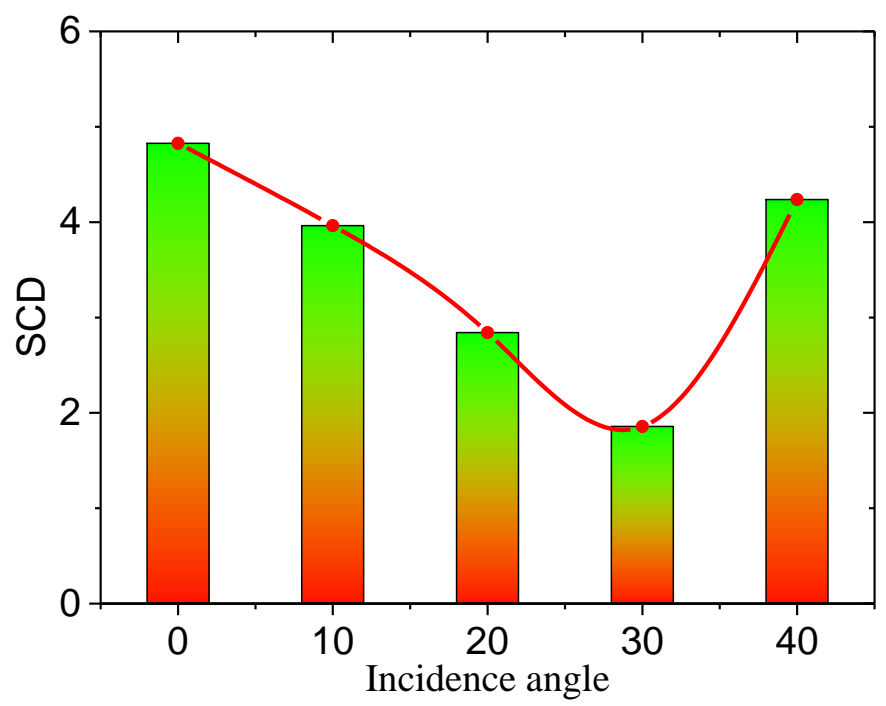

Figure 12. SCD of the pressure signal time series at different incidence angles.

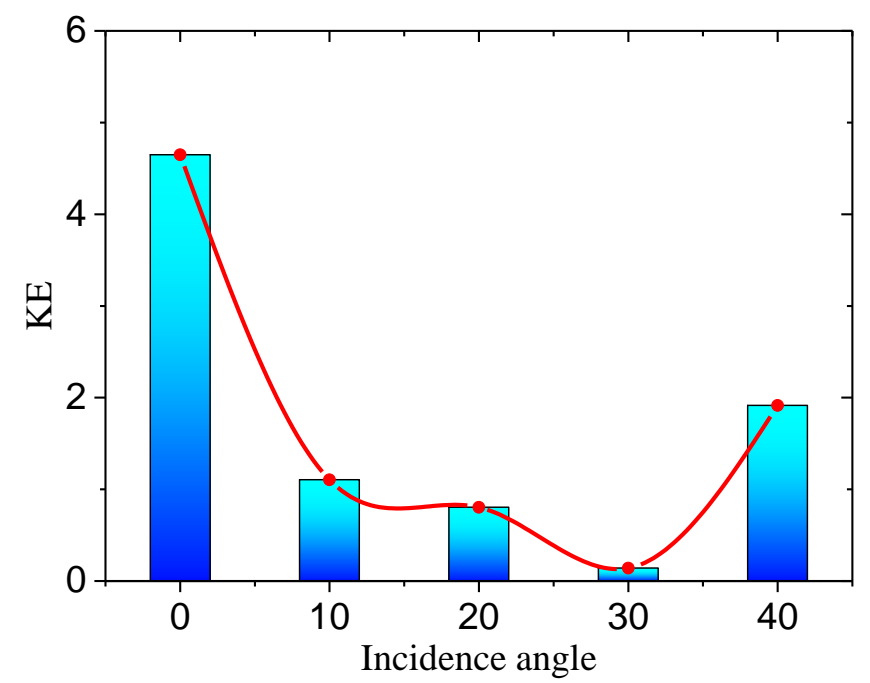

Figure 13. KE of the pressure signal time series at different incidence angles. 


\subsubsection{Analysis of the PSD Features}

In order to reveal the inherent features of the flow field with different incidence angles, the pressure coefficient contours are shown in Figure 14. From Figure 14, we can see that the influence of the incidence angle on the flow structure is obvious. The number and shedding frequency of the vortex change with the incidence angle. The results suggest that the PSD may be a distinguishing feature. Therefore, the fast Fourier transform (FFT) is performed on the signal sequence, and the PSD with different incidence angles is shown in Figure 15.
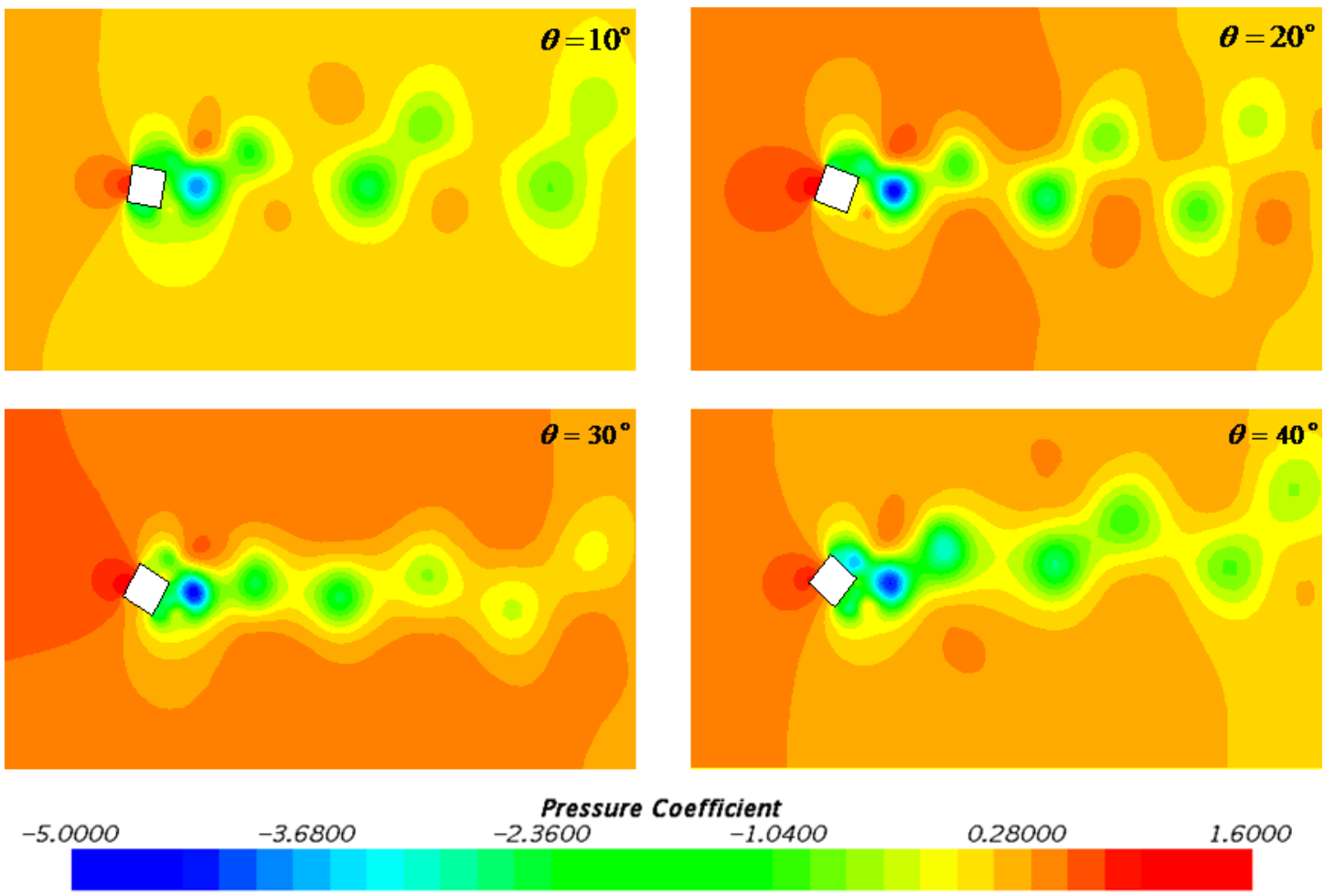

Figure 14. Pressure coefficient contours of the flow field around the square target at different incidence angles.

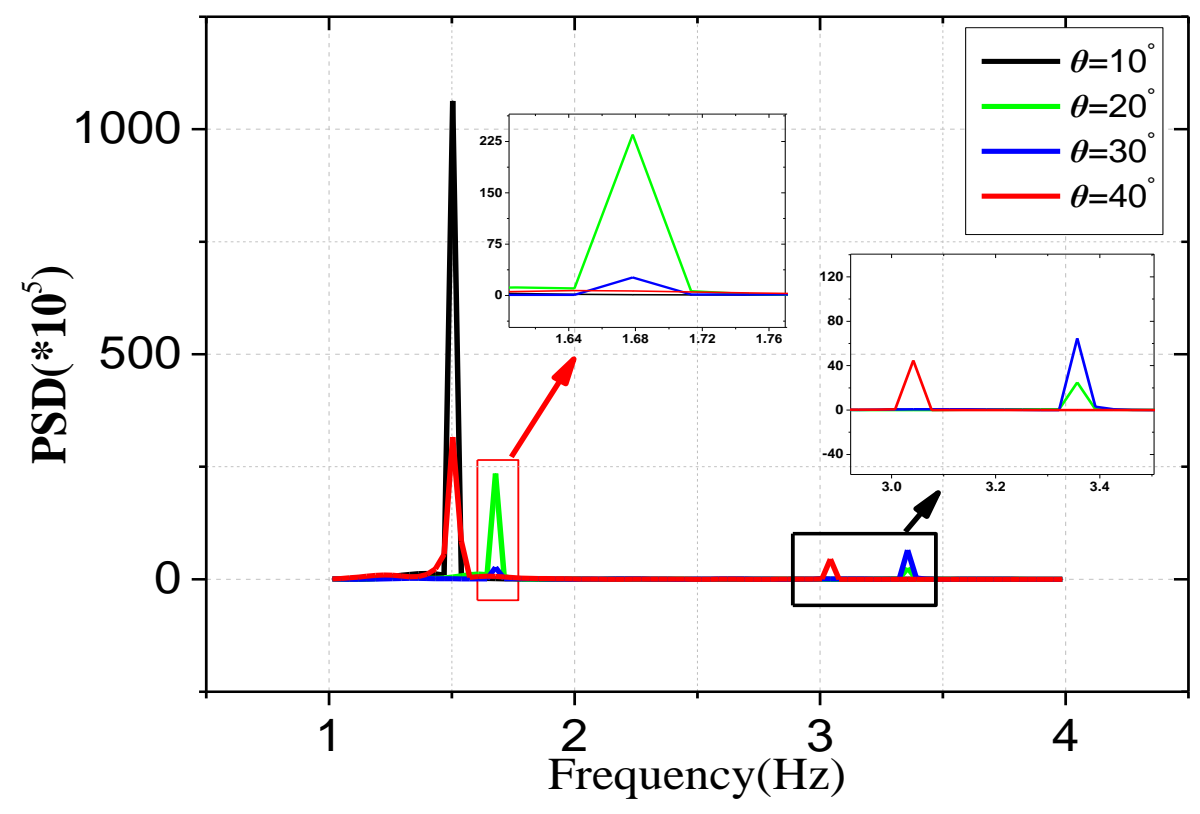

Figure 15. Effects of incidence angles of the square target on the power spectrum density. 
As seen in Figure 15, when the incidence angle is $10^{\circ}$, there is one obvious crest at the position of $f_{10}=1.5 \mathrm{~Hz}$. At other incidence angles, there are two crests in the curve. The positions of the two crests are similar when the incidence angles are $20^{\circ}$ and $30^{\circ}$. However, when the incidence angle is $20^{\circ}$, the first crest $\left(f_{20,1}=f_{30,1}=1.68 \mathrm{~Hz}\right)$ is stronger than the second crest $\left(f_{20,2}=f_{30,2}=3.36 \mathrm{~Hz}\right)$, which is opposite to the situation of $\theta=30^{\circ}$. The first crest of $\theta=40^{\circ}$ coincides with $f_{10}$, but the intensity is lower than that of $f_{10}$. Another crest is at the position of $f_{40,2}=3.04 \mathrm{~Hz}$.

The results are exciting, because the PSD values at different incidence angles have no overlap. Therefore, the number, amplitude, and position of wave crests on the PSD curves can be used as the distinguishing features to recognize the incidence angles of one target.

\section{Numerical Experiments and Discussion}

The support vector machine (SVM) was developed from the theory of structural risk minimization and designed for linear classifiers with the linear function $\omega^{T} \cdot \bar{x}+b$, where $\omega$ is the weight vector and $b$ is the shift of the separating hyperplane. The margin between the samples and the separating hyperplane is $2 /\|\omega\|$ when the labels are +1 or -1 . Therefore, the goal of the SVM is to get the minimum value of $\|\omega\|^{2}$. A Lagrangian equation $L(\omega, b, \alpha)$ was built to finish the goal, which is shown in Equation (16). Based on Equation (17), interesting results are shown in Equation (18).

$$
\begin{gathered}
L(\vec{\omega}, b, \alpha)=\frac{1}{2}\|\vec{\omega}\|^{2}-\sum_{i=1}^{n} \alpha_{i}\left[y_{i}\left(\vec{\omega} \cdot \vec{x}_{i}+b\right)-1\right], \\
\left\{\begin{array}{l}
\frac{\partial L}{\partial \vec{\omega}}=0 \Rightarrow \vec{\omega}=\sum_{i=1}^{n} \alpha_{i} y_{i} x_{i} \\
\frac{\partial L}{\partial b}=0 \Rightarrow \sum_{i=1}^{n} \alpha_{i} y_{i}=0 \\
\frac{\partial L}{\partial \alpha}=0 \Rightarrow \alpha_{i}\left[y_{i}\left(\vec{\omega} \cdot \vec{x}_{i}+b\right)-1\right]=0
\end{array}\right. \\
\left\{\begin{array}{l}
\vec{\omega}_{\min }=\sum_{i=1}^{n} \alpha_{i}^{*} y_{i} \vec{x}_{i} \\
f(x)=\operatorname{sgn}[(\vec{\omega} \cdot \vec{x})+b]=\operatorname{sgn}\left[\sum_{i=1}^{n} \alpha_{i}^{*} y_{i}\left(\vec{x}_{i} \cdot \vec{x}\right)+b^{*}\right]
\end{array}\right.
\end{gathered}
$$

where $y_{i}$ is the corresponding label, $x_{i}$ is a support vector when $0<\alpha_{i}<C$, and $C$ is the penalty factor.

For a non-linear classification, the SVM is sufficient with the help of the kernel trick [49]. Compared with the linear SVM classifier, a higher dimensional feature space $\Psi$ is used, which is nonlinearly related to the input space, $\Phi: \mathfrak{R}^{n} \rightarrow \Psi$. In the feature space $\Psi$, the Euclidean dot-products $(\vec{x} \cdot \vec{y})$ are transformed to $(\Phi(\vec{x}) \cdot \Phi(\vec{y}))$ based on a kernel function, which is a polynomial kernel in this paper and is shown in Equation (19).

$$
k\left(x, y_{i}\right)=\left(g \times\left(\vec{x} \cdot \vec{y}_{i}\right)+r\right)^{q},
$$

where $g$ and $r$ are the parameters, which are 1 in this paper, and $q$ is the order of the kernel function.

The grid search algorithm is used to find the optimal $C$ and $q$. Five-fold cross-validation is used firstly to determine the half-optimal $C_{1 / 2}$ in $\left[2^{-6}, 2^{6}\right]$ and $q_{1 / 2}$ in $\left[2^{-10}, 2^{10}\right]$; then 10 -fold cross-validation is used in $\left[\frac{C_{1 / 2}}{4}, 4 C_{1 / 2}\right]$ and $\left[\frac{q_{1 / 2}}{4}, 4 q_{1 / 2}\right]$ to find the optimal $C_{\text {opt }}$ and $q_{\text {opt }}$.

Three kinds of target with different shapes are considered, including a circular target, triangular target, and square target. The pressure signal on the virtual LLS is selected to build the classification datasets $\Gamma_{3000 \times 11}=\left(\left\{\Gamma_{c}\right\}_{3000 \times 1},\left\{\Gamma_{t}\right\}_{3000 \times 5},\left\{\Gamma_{s}\right\}_{3000 \times 5}\right)$, and five datasets $\left\{\left(\eta_{0}, \eta_{15}, \eta_{30}, \eta_{45}, \eta_{60}\right)\right\}$ and $\left\{\left(\gamma_{0}, \gamma_{10}, \gamma_{20}, \gamma_{30}, \gamma_{40}\right)\right\}$ are included in $\left\{\Gamma_{t}\right\}$ and $\left\{\Gamma_{s}\right\}$. The classification strategy is shown in Figure 16. 


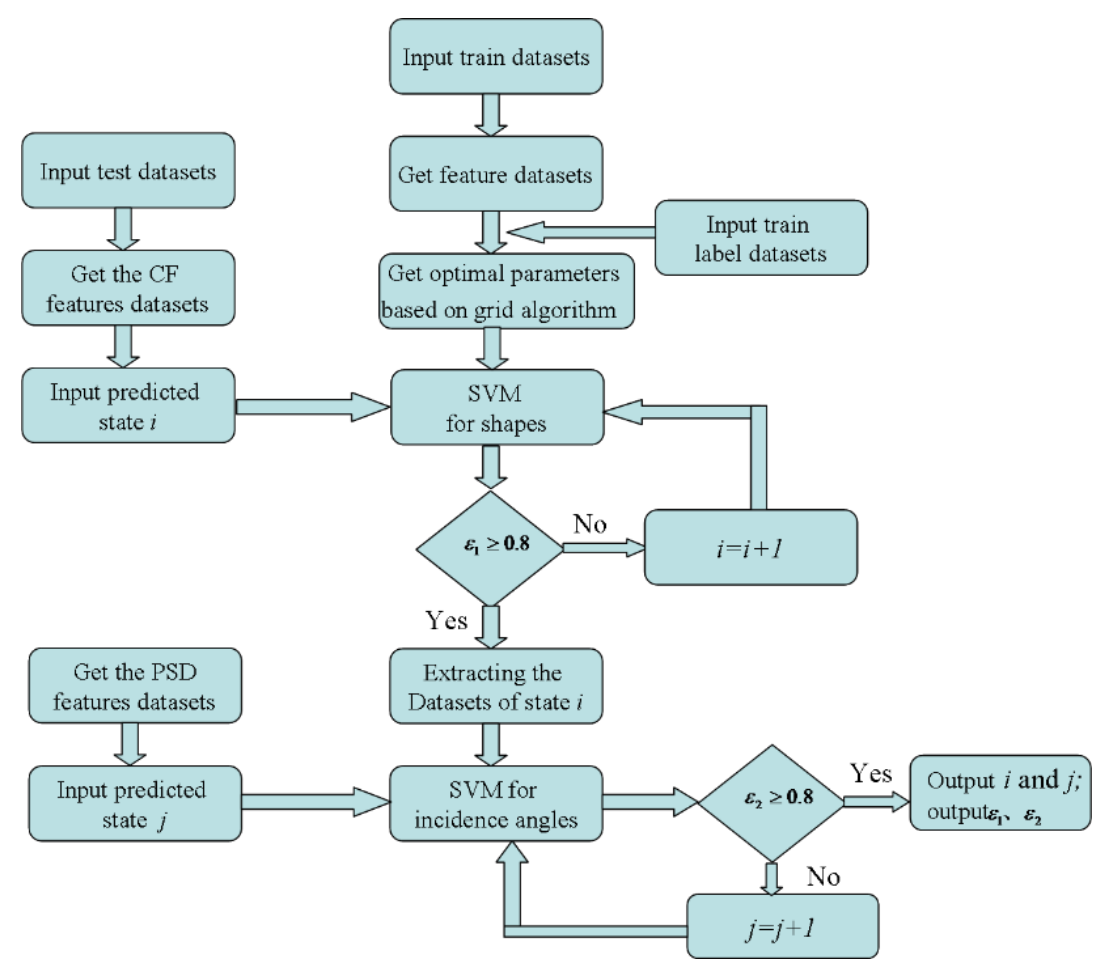

Figure 16. Workflow of the proposed classification strategy.

The chaotic features are used firstly to classify the shapes of targets based on the above SVM; then, the PSD features are selected to classify the incidence angles [50]. For the first step, $\Gamma$ is used as the training data, and a single individual from each type of signal is selected as the testing data. For convenient comparison, $\Gamma_{c}$ is set as label 1, and the other two groups, $\Gamma_{t}$ and $\Gamma_{s}$, are set as label -1 . The central mark is the recognition rate (RR). If the RR is larger than 0.8 , this implies that the testing data is $\Gamma_{c}$; if not, it implies that the testing data belongs to $\Gamma_{t}$ or $\Gamma_{s}$. Then, the same process is carried out again until the category is decided. The classification of incidence angles is the same as the above procedure. Table 2 lists the extracted RR and corresponding averaged value (AR). From the table, it can be seen that the method gets a recognition rate of $96.73 \%$ for shape recognition, and a recognition rate of $92.48 \%$ for incidence angle recognition. Therefore, the proposed method can achieve better performance under these features.

Table 2. Classification results of the support vector machine (SVM) method based on chaotic features (CF)-power spectrum density (PSD) features.

\begin{tabular}{|c|c|c|c|c|c|c|c|c|c|c|c|c|}
\hline \multirow{2}{*}{$\mathrm{RR} / \%$} & \multirow{2}{*}{ Circular } & \multicolumn{5}{|c|}{ Triangular } & \multicolumn{5}{|c|}{ Square } & \multirow{2}{*}{$\mathrm{AR} / \%$} \\
\hline & & $0^{\circ}$ & $15^{\circ}$ & $30^{\circ}$ & $45^{\circ}$ & $60^{\circ}$ & $0^{\circ}$ & $10^{\circ}$ & $20^{\circ}$ & $30^{\circ}$ & $40^{\circ}$ & \\
\hline$\varepsilon_{1}$ & 100 & & & 90.2 & & & & & 100 & & & 96.73 \\
\hline$\varepsilon_{2}$ & 1 & 95.4 & 89.7 & 92.3 & 95.7 & 93.8 & 94.6 & 88.5 & 87.3 & 91.7 & 95.8 & 92.48 \\
\hline
\end{tabular}

\section{Conclusions}

The flow features were analyzed in the framework of chaotic theory, and a new strategy for underwater target classification using these features was presented, which includes the CF and PSD. A two-step SVM was built based on the compounded features CF-PSD, and it was used to classify the shapes and incidence angles of different targets. Some conclusions were obtained from the results, which are as follows: 
1. The flow field around the circular target is periodic, and the systems around the triangle target and square target are chaotic systems. The chaotic features of the pressure signal time series in different flow fields have no overlap, which implies that CF can be used to classify the shapes of targets.

2. The relationship between CF and incidence angles is not monotonic, and CF cannot be used to recognize the incidence angles of square targets. However, the number, amplitude, and position of wave crests on the PSD curves have no overlap, and PSD is able to distinguish the incidence angles.

3. A two-step SVM with a polynomial kernel was built. It can achieve better performance under the compounded features CF-PSD and has a recognition rate of $96.73 \%$ for shape classification and a recognition rate of $92.48 \%$ for incidence angle recognition.

Author Contributions: Conceptualization, X.L. and J.W.; methodology, X.L.; software, X.L. and Q.Q.; validation, J.W., X.L. and Q.Q.; formal analysis, X.L. and Q.Q.; investigation, X.L. and J.W.; resources, J.W.; data curation, X.L.; writing-original draft preparation, X.X.; writing—review and editing, J.W. and Q.Q.; visualization, X.L. and Q.Q.; supervision, J.W.; project administration, X.L.; funding acquisition, J.W. All authors have read and agreed to the published version of the manuscript.

Funding: This research was funded by the NATIONAL NATURAL AND SCIENCE FOUNDATION OF HEBEI, grant number E2018202259.

Acknowledgments: The authors would like to thank Xiaoming Wang and Haitao Liu, who provided insight and expertise that greatly assisted in the writing of this manuscript.

Conflicts of Interest: The authors declare no conflict of interest. The funders had no role in the design of the study; in the collection, analyses, or interpretation of data; in the writing of the manuscript, or in the decision to publish the results.

\section{References}

1. Myint, M.; Yonemori, K.; Lwin, K.N.; Yanou, A.; Minami, M. Dual-eyes Vision-based Docking System for Autonomous Underwater Vehicle: An Approach and Experiments. J. Intell. Robot. Syst. 2017, 92, 159-186. [CrossRef]

2. Jebelli, A.; Yagoub, M.C.E.; Dhilon, B.S. Design and Control of a Self-balancing Autonomous Underwater Vehicle with Vision and Detection Capabilities. J. Mar. Sci. Res. Dev. 2018, 8, 1000245.

3. Isakson, M.J. Through-the-sensor Sediment Characterization from Autonomous Underwater Vehicle Sonar Systems. J. Acoust. Soc. Am. 2017, 141, 4042. [CrossRef]

4. King, P.; Anstey, B.; Vardy, A. Sonar Image Registration for Localization of an Underwater Vehicle. J. Ocean Technol. 2017, 12, 68-90.

5. Emberton, S.; Chittka, L.; Cavallaro, A. Underwater Image and Video Dehazing with Pure Haze Region Segmentation. Comput. Vis. Image Und. 2017, 168, 145-156. [CrossRef]

6. Li, C.; Guo, J. Underwater Image Enhancement by Dehazing and Color Correction. J. Electron. Imaging 2015, 24, 033023. [CrossRef]

7. Sato, M.; Asaoka, R.; Nakae, M.; Sasaki, K. The Lateral Line System and Its Innervation in Lateolabrax Japonicus (Percoidei Incertae Sedis) and Two Apogonids (Apogonidae), with Special Reference to Superficial Neuromasts (Teleostei: Percomorpha). Ichthyol. Res. 2017, 64, 308-330. [CrossRef]

8. McHenry, M.J.; Feitl, K.E.; Strother, J.A.; Trump, W.J.V. Larval Zebrafish Rapidly Sense the Water Flow of a Predator's strike. Biol. Lett. 2009, 5, 477-479. [CrossRef]

9. Karine, F.; Eric, P.; Christophe, B.; Vandewalle, N.; Vandewalle, P. Fish Lateral System is Required for Accurate Control of Shoaling Behaviour. Anim. Behav. 2010, 79, 679-687.

10. Hildur, K.; Cole, Z.; Dhananjay, B.; Palsson, E.; Nogare, D.D.; Chitnis, A.B.; Keshet, L.E. Polarization and Migration in the Zebrafish Posterior Lateral Line System. PLOS Comput. Biol. 2017, 13, 1005451.

11. Fernandez, V.I. Performance Analysis for Lateral Line Inspired Sensor Arrays. Ph.D. Thesis, Massachusetts Institute of Technology and Woods Hole Oceanographic Institution, Boston, MA, USA, 2011.

12. Jiang, Y.G.; Ma, Z.Q.; Fu, J.C.; Zhang, D.Y. Development of a Flexible Artificial Lateral Line Canal System for Hydrodynamic Pressure Detection. Sensors 2017, 17, 1220. [CrossRef] [PubMed] 
13. Han, Z.W.; Liu, L.P.; Wang, K.J.; Song, H.L.; Chen, D.B.; Wang, Z.; Niu, S.C.; Zhang, J.Q.; Ren, L.Q. Artificial Hair-like Sensors Inspired from Nature: A Review. J. Bionic Eng. 2018, 15, 409-434. [CrossRef]

14. Yang, Y.C.; Nguyen, N.; Chen, N.N.; Lockwood, M.; Tucker, C.; Hui, H.; Bleckmann, H.; Liu, C.; Jones, D.L. Artificial Lateral Line with Biomimetic Neuromasts to Emulate Fish Sensing. Bioinspir. Biomim. 2010, 5 , 016001. [CrossRef] [PubMed]

15. Liu, G.J.; Wang, M.M.; Wang, A.Y.; Wang, S.R.; Yang, T.T.; Malekian, R.; Li, Z.X. Research on Flow Field Perception Based on Artificial Lateral Line Sensor System. Sensors 2018, 18, 838. [CrossRef] [PubMed]

16. Zhou, H.; Hu, T.J.; Low, K.H.; Shen, L.C.; Ma, Z.W.; Wang, G.M.; Xu, H.J. Bio-inspired Flow Sensing and Prediction for Fish-like Undulating Locomotion: A CFD-aided Approach. J. Bionic Eng. 2015, 12, 406-417. [CrossRef]

17. Boulogne, L.H.; Wolf, B.J.; Wiering, M.A.; Netten, S.M.V. Performance of Neural Networks for Localizing Moving Objects with an Artificial Lateral Line. Bioinspir. Biomim. 2017, 12, 056009. [CrossRef]

18. Sarlo, R.; Najem, J.S.; Leo, D.J. Flow Field Sensing with Bio-inspired Artificial Hair Cell Arrays. Sens. Actuator B Chem. 2016, 236, 805-814. [CrossRef]

19. Dusek, J.E.; Triantafyllou, M.S.; Lang, J.H. Piezoresistive Foam Sensor Arrays for Marine Applications. Sens. Actuator A Phys. 2016, 248, 173-183. [CrossRef]

20. Delamare, J.; Sanders, R.G.P.; Krijnen, G.J.M. 3D Printed Biomimetic Whisker-based Sensor with Co-planar Capacitive Sensing. In Proceedings of the 2016 IEEE International Conference on Sensors, Orlando, FL, USA, 30 October-3 November 2016.

21. Zhu, P.; Ma, B.; Jiang, C.; Deng, J.; Wang, Y. Improved Sensitivity of Micro Thermal Sensor for Underwater Wall Shear Stress Measurement. Microsyst. Technol. 2015, 21, 785-789. [CrossRef]

22. Djapic, V.; Dong, W.J.; Bulsara, A.; Anderson, G. Challenges in Underwater Navigation: Exploring Magnetic Sensors Anomaly Sensing and Navigation. In Proceedings of the 2016 IEEE International Conference on Sensors Applications Symposium, Zadar, Croatia, 13-15 April 2015.

23. Salmanpour, M.S.; Sharif, K.Z.; Aliabadi, M. Impact Damage Localisation with Piezoelectric Sensors under Operational and Environmental Conditions. Sensors 2017, 17, E1178. [CrossRef]

24. Campos, R.; Gracias, N.; Ridao, P. Underwater Multi-vehicle Trajectory Alignment and Mapping Using Acoustic and Optical Constraints. Sensors 2016, 16, 387. [CrossRef] [PubMed]

25. Abdulsadda, A.T.; Tan, X. Nonlinear Estimation-based Dipole Source Localization for Artificial Lateral Line Systems. Bioinspir. Biomim. 2013, 8, 026005. [CrossRef]

26. Abdulsadda, A.T.; Tan, X. An Artificial Lateral Line System Using IPMC Sensor Arrays. Inter. J. Smart Nano Mater. 2012, 3, 226-242. [CrossRef]

27. Dagamseh, A.M.K.; Lammerink, T.S.J.; Kolster, M.L.; Bruinink, C.M.; Wiegerink, R.J.; Krijnen, G.J.M. Dipole-source Localization Using Biomimetic Flow-sensor Arrays Positioned as Lateral-line System. Sens. Actuator A Phys. 2010, 162, 355-360. [CrossRef]

28. Salumae, T.; Kruusmaa, M. Flow-relative Control of an Underwater Robot. Proc. R. Soc. A Math. Phys. 2013, 469, 1-19. [CrossRef]

29. Venturelli, R.; Akanyeti, O.; Visentin, F.; Jezov, J.; Chambers, L.; Toming, G.; Brown, J.; Kruusmaa, M.; Megill, W.M.; Fiorini, P. Hydrodynamic Pressure Sensing with an Artificial Lateral Line in Steady and Unsteady Flows. Bioinsper. Biomim. 2012, 7, 036004. [CrossRef]

30. Abdulsadda, A.T.; Tan, X. Underwater Tracking of a Moving Dipole Source Using an Artificial Lateral Line: Algorithm and Experimental Validation with Ionic Polymer-metal Composite Flow Sensors. Smart Mater. Struct. 2013, 22, 045010. [CrossRef]

31. Humphrey, J.A. Drag Force Acting on a Neuromast in the Fish Lateral Line Trunk Canal. II. Analytical Modelling of Parameter Dependencies. J. R. Soc. Interface 2009, 6, 641-653. [CrossRef]

32. Kumar, P.S.; Vendhan, C.P.; Krishnankutty, P. Study of Water Wave Diffraction Around Cylinders Using a Finite-element Model of Fully Nonlinear Potential Flow Theory. Ships Offshore Struct. 2017, 12, $276-289$. [CrossRef]

33. Fuentes-Perez, J.F.; Uhtan, J.A.; Baeza, R.C.; Musall, M.; Toming, G.; Muhammad, N. Current Velocity Estimation Using a Lateral Line Probe. Ecol. Eng. 2015, 85, 296-300. [CrossRef]

34. Xu, D.; Lv, Z.; Liu, J.; Wang, J. A novel artificial lateral line sensing system of robotic fish based on BP neural network. In Proceedings of the 2017 12th IEEE Conference on Industrial Electronics and Applications, Siem Reap, Cambodia, 18-20 June 2017. 
35. Varon, E.; Eulalie, Y.; Edwige, S.; Gilotte, P.; Aider, J.L. Chaotic Dynamics of Large-scale Structures in a Turbulent Wake. Phys. Rev. Fluids 2017, 2, 034604. [CrossRef]

36. Narayanan, S.; Gunaratne, G.H.; Hussain, F. A Dynamical Systems Approach to the Control of Chaotic Dynamics in a Spatiotemporal Jet Flow. Chaos 2013, 23, 033133. [CrossRef]

37. Lin, X.H.; Wu, J.G.; Qin, Q. A Novel Obstacle Localization Method for an Underwater Robot Based on the Flow Field. J. Mar. Sci. Eng. 2019, 7, 437. [CrossRef]

38. Bao, Y.; Zhou, D.; Zhao, Y.J. A Two Step Taylor-characteristic-based Galerkin Method for Incompressible Flows and Its Application to Flow over Triangular Cylinder with Different Incidence Angles. Int. J. Numer. Meth. Fluids 2010, 62, 1181-1208. [CrossRef]

39. Zhao, X.Z.; Cheng, D.; Zhang, D.K.; Hu, Z.J. Numerical Study of Low-Reynolds-number Flow Past Two Tandem Square Cylinders with Varying Incident Angles of the Downstream One Using a CIP-based Model. Ocean Eng. 2016, 121, 414-421. [CrossRef]

40. Yoon, D.H.; Yang, K.S.; Choi, C.B. Flow Past a Square Cylinder with an Angle of Incidence. Phys. Fluids 2010, 22, 043603. [CrossRef]

41. Takens, F. Detecting Strange Attractors in Turbulence. In Dynamical Systems and Turbulence; Springer: Berlin, Germany, 1981; pp. 154-196.

42. Kim, H.S.; Eykholt, R.; Salas, J.D. Nonlinear Dynamics, Delay times, and Embedding Windows. Physica D 1999, 127, 48-60. [CrossRef]

43. Song, W.Q.; Deng, S.; Yang, J.; Cheng, Q. Tool Wear Detection Based on Duffing-Holmes Oscillator. Math. Probl. Eng. 2008, 2008, 510406. [CrossRef]

44. Li, Q.; Liang, S.Y.; Yang, J.; Li, B. Long Range Dependence Prognostics for Bearing Vibration Intensity Chaotic Time Series. Entropy 2016, 18, 23. [CrossRef]

45. Wolf, A.; Swift, J.B.; Swinney, H.L.; Vastano, J.A. Determining Lyapunov Exponents from a Time Series. Physica D 1985, 16, 285-317. [CrossRef]

46. Vastano, J.A.; Kostelich, E.J. Comparison of algorithms for determining Lyapunov exponents from experimental data. In Dimensions and Entropies in Chaotic Systems; Gottfried, M.K., Ed.; Springer: Berlin/Heidelberg, Germany, 1986; pp. 100-107.

47. Grassberger, P.; Procaccia, I. Dimensions and Entropies of Strange Attractors from a Fluctuating Dynamics Approach. Physica D 1984, 13, 34-54. [CrossRef]

48. Grassberger, P.; Procaccia, I. Measuring the Strangeness of Strange Attractors. Physica D 1983, 9, $189-208$. [CrossRef]

49. Wang, S.; Zeng, X. Robust Underwater Noise Targets Classification Using Auditory Inspired Time-frequency Analysis. Appl. Acoust. 2014, 78, 68-76. [CrossRef]

50. Tang, F.; Adam, L.; Si, B. Group Feature Selection with Multiclass Support Vector Machine. Neurocomputing 2018, 317, 42-49. [CrossRef]

(C) 2020 by the authors. Licensee MDPI, Basel, Switzerland. This article is an open access article distributed under the terms and conditions of the Creative Commons Attribution (CC BY) license (http://creativecommons.org/licenses/by/4.0/). 